Article

\title{
Carbohydrate Electrolyte Solutions Enhance Endurance Capacity in Active Females
}

\author{
Feng-Hua Sun ${ }^{1}$, Stephen Heung-Sang Wong ${ }^{2, *}$, Shi-Hui Chen ${ }^{1}$ and \\ Tsz-Chun Poon ${ }^{2}$
}

1 Department of Health and Physical Education, Hong Kong Institute of Education, Rm D4-2/F-13, 10 Lo Ping Road, Tai Po, Hong Kong, 00852, China; E-Mails: fhsun@ied.edu.hk (F.-H.S.); shchen@ied.edu.hk (S.-H.C.)

2 Department of Sports Science and Physical Education, Chinese University of Hong Kong, G08, Kwok Sports Building, Chinese University of Hong Kong, Shatin, Hong Kong, 00852, China; E-Mail: ericpoontc@gmail.com

* Author to whom correspondence should be addressed; E-Mail: hsswong@cuhk.edu.hk; Tel.: +00-852-3943-6095; Fax: +00-852-2603-5781.

Received: 6 February 2015 / Accepted: 11 May 2015 / Published: 15 May 2015

\begin{abstract}
The purpose of the present study was to investigate the effects of supplementation with a carbohydrate-electrolyte solution (CES) in active females during a prolonged session of submaximal running to exhaustion. Eight healthy active females volunteered to perform a session of open-ended running to exhaustion at $70 \%$ of their maximal oxygen consumption on a treadmill during the follicular phase of their menstrual cycle on two occasions. During each run, the subjects consumed either $3 \mathrm{~mL} \cdot \mathrm{kg}^{-1}$ body mass of a $6 \%$ CES or a placebo drink (PL) every $20 \mathrm{~min}$ during exercise. The trials were administered in a randomized double-blind, cross-over design. During the run, the subjects ingested similar volumes of fluid in two trials (CES: $644 \pm 75 \mathrm{~mL} v$. PL: $593 \pm 66 \mathrm{~mL}, p>0.05$ ). The time to exhaustion was $16 \%$ longer during the CES trial $(106.2 \pm 9.4 \mathrm{~min})$ than during the PL trial $(91.6 \pm 5.9 \mathrm{~min})(p<0.05)$. At 45 min during exercise, the plasma glucose concentration in the CES trial was higher than that in PL trial. No differences were observed in the plasma lactate level, respiratory exchange ratio, heart rate, perceived rate of exertion, sensation of thirst, or abdominal discomfort between the two trials $(p>0.05)$. The results of the present study confirm that CES supplementation improves the moderate intensity endurance capacity of active females during the follicular phases of the menstrual cycle. However, the exogenous oxidation of carbohydrate does not seem to explain the improved capacity after CES supplementation.
\end{abstract}


Keywords: running; exercise to exhaustion; follicular phase

\section{Introduction}

The successful completion of an exhaustive endurance exercise depends on numerous factors. The availability of substrate provision, muscle glycogen storage, and hydration status are among the most commonly acknowledged factors that influence fatigue [1,2]. Fatigue during prolonged submaximal exercise at a moderate to high intensity equivalent to $65 \%$ to $85 \%$ of the maximal oxygen consumption $\left(\mathrm{VO}_{2 \max }\right)$ is to a large extent the result of the depletion of muscle glycogen in skeletal muscle, and a reduction in the blood glucose concentration $[3,4]$. Therefore, in recent years the potential role played by the ingestion of carbohydrates $(\mathrm{CHO})$ during exercise has been extensively investigated, and recommendations for $\mathrm{CHO}$ ingestion during endurance exercise have been made [5].

Several systematic reviews have recently summarized the effects of $\mathrm{CHO}$ ingestion during exercise on endurance performance [6-8]. These reviews have concluded that $\mathrm{CHO}$ ingestion plays a positive role during endurance exercise, and the potential mechanisms involved have been discussed. However, previous studies show a strong gender bias in that trained males were usually recruited as the participants [6]. Almost all of the research findings from male participants have been indiscriminately generalized and applied to female athletes. Relatively little attention has been directed toward gender differences in the effects of $\mathrm{CHO}$ ingestion during exercise. To our knowledge, only three studies have exclusively recruited female participants [9-11]. In general, female oxidize more lipids, fewer proteins, and fewer total CHO than male during endurance exercise [12]. The lower use of glycogen in skeletal muscle and lower production of hepatic glucose have also been found in female than in male [12]. Consequently, it is possible that $\mathrm{CHO}$ supplementation during exercise may have different effects in female than in male. There is thus an overwhelming need for more well-controlled experimental studies in female.

Although the form of $\mathrm{CHO}$ (liquid, semi-liquid, or solid) is not regarded to be very important when considering the potential ergogenic effects of $\mathrm{CHO}$ ingestion during exercise [13,14], CHO beverages are usually used. This may be mainly due to issues of hydration. Although the depletion of CHO reserves is believed to be the primary cause of fatigue in prolonged exhaustive exercise, other concurrent factors exist to limit human performance. One of the physiological perturbations that cause early fatigue is dehydration [2,15]. Fluid consumption throughout prolonged exercise has been shown to decrease dehydration and attenuate its associated effects on thermoregulation, cardiovascular functions, and exercise performance [16]. Because the ingestion of plain water may decrease plasma osmolality and sodium concentration, so as to stimulate the production of urine and reduce the urge to drink [17], it may not be appropriate for consumption during endurance exercise. A small amount of electrolytes added to the beverage could improve its palatability and encourage sufficient fluid replacement. As recommended by the American College of Sports Medicine, and supported by thoroughly investigated research findings, the regular ingestion of 150 to $250 \mathrm{~mL}$ of CHO-electrolyte solution (CES) every 15 to $20 \mathrm{~min}$ during moderate intensity exercise is an appropriate choice [2].

Therefore, the purpose of the current study was to investigate the influence of CES ingestion during prolonged submaximal running on the endurance capacity of recreationally active females. 


\section{Experimental Section}

\subsection{Subjects}

Eight healthy, non-smoking, recreationally active female subjects were recruited from the university population and athletics clubs in Hong Kong. Their age, height, weight, percentage of body fat, and $\mathrm{VO}_{2 \max }($ mean $\pm \mathrm{SEM})$ were $28.3 \pm 1.5$ years, $155.2 \pm 1.4 \mathrm{~cm}, 47.8 \pm 0.7 \mathrm{~kg}, 16.2 \% \pm 0.8 \%$, and $48.3 \pm 2.1 \mathrm{~mL} \cdot \mathrm{kg}^{-1} \cdot \mathrm{min}^{-1}$, respectively. Each subject participated regularly in various forms of endurance training (at least three sessions per week with more than $30 \mathrm{~min}$ in each session) and was considered recreationally active. A statement of written informed consent was obtained after the nature of the experimental procedures and the potential risks and benefits were thoroughly explained. The subjects also completed questionnaires about their medical histories and general habits. None of the subjects had an adverse medical history, major muscular condition or injury that would impede moderate intensity endurance running. In addition, the successful completion of at least one hour of endurance running at $70 \%$ of $\mathrm{VO}_{2 \max }$ was a minimum requirement for inclusion in the investigation. The procedure was approved by the Ethics Committee of the Chinese University of Hong Kong.

\subsection{Preliminary Measurements}

Two preliminary tests, the $\mathrm{VO}_{2}$ max test and the $\mathrm{VO}_{2}$-Speed test, were conducted before the two main trials were undertaken. The $\mathrm{VO}_{2 \max }$ was determined for each subject by means of a continuous, incremental, graded uphill treadmill running test (Quinton, Model 24-72) to volitional exhaustion, as described elsewhere [18]. $\mathrm{VO}_{2 \max }$ was reached when the following criteria were met: (a) a plateau of $\mathrm{VO}_{2}$ with increasing work rate; (b) a respiratory exchange ratio (RER) of greater than 1.15; and (c) a heart rate (HR) within 5 beats/min of the age-predicted maximal HR. The relationship between $\mathrm{VO}_{2}$ and submaximal running speed on a level treadmill for each subject was determined in a 16-min incremental submaximal running test. Four speeds were chosen with reference to each subject's training status and set between $60 \%$ and $70 \%$ of $\mathrm{VO}_{2 \max }$. The subjects ran for $4 \mathrm{~min}$ at each speed. Expired air samples were collected using the Douglas bag method during the last minute of each 4 min period and analyzed. Each subject's HR and rate of perceived exertion (RPE) were also monitored and recorded throughout the run. Running speeds equivalent to $70 \%$ of each individual's $\mathrm{VO}_{2 \max }$ were determined from the results of these two tests.

One week before the first main trial, a 60 -min familiarization treadmill run was also conducted to verify, and if necessary adjust, the running speed for the main trials. During this run, all of the procedures were standardized and were identical to those used during the main trials. This process enabled the subjects to be fully familiarized with all of the procedures performed and the measurements made during the main trials.

\subsection{Experimental Procedures}

Baseline analyses of the nutritional content of each subject's normal diet were obtained on the basis of their 3-day weighed food record diaries before the main experimental trials. The amount, weight and frequency of all food and fluid consumed, and the ingestion of any extra vitamin or mineral supplements 
were recorded each day. The dietary records were analyzed with computer software (Food Processor 10.5, ESHA, Salem, Oregon). The subjects were instructed to repeat the same diet during the 3 days before each subsequent trial. The subjects were asked to maintain their current level of training throughout the study and to incorporate the experimental test into their training schedule as a "hard work out". Two days before each test, the subjects were required to refrain from strenuous exercise to exclude any residual effects of fatigue from prior exercise on the experimental treatments. The subjects were also asked to avoid any foods or beverages that might induce diuresis during the 24-h period before the experiments. To increase the likelihood of euhydration before each testing session, the subjects were instructed to ingest approximately $500 \mathrm{ml}$ of water in the evening before the tests.

Two open-ended runs to exhaustion at $70 \%$ of $\mathrm{VO}_{2 \max }$ were completed on a level treadmill approximately 1 month apart during the follicular phase of the participants' menstrual cycle. The experiments were conducted on the day during which the menstrual bleeding period ended because the levels of both estradiol and progesterone were likely to be low. On each occasion, the subjects were required to consume either a diluted CES $\left(19 \mathrm{mEq} \mathrm{Na}{ }^{+}\right.$and $6 \% \mathrm{CHO}, \mathrm{CES}$ ) or a placebo (glucose- and electrolyte-free artificially sweetened drink, PL) at every $20 \mathrm{~min}\left(3 \mathrm{~mL} \cdot \mathrm{kg}^{-1}\right.$ body mass, BM). The experimental drinks were similar in color, texture, taste, and temperature. The study was conducted in the Exercise Physiology Laboratory under similar neutral environmental conditions (CES vs. PL: temperature, $20.3{ }^{\circ} \mathrm{C} \pm 0.7{ }^{\circ} \mathrm{C}$ vs. $21.3{ }^{\circ} \mathrm{C} \pm 0.7{ }^{\circ} \mathrm{C}$; relative humidity, $60.6 \% \pm 1.2 \%$ vs. $64.4 \% \pm 2.3 \%$ ). The experiments were administered in a double-blind cross-over design in a random order.

On the day of the experiment, the subjects reported to the laboratory after an overnight fast of at least $10 \mathrm{~h}$. On arrival, the subjects were required to rest for about $15 \mathrm{~min}$ and drink $250 \mathrm{~mL}$ of water. The subject's nude body weight was measured before and after each run. A HR monitor (Sports Tester PE3000, Polar Electro, Finland) was attached to each subject to monitor the HR during the treadmill test. Expired gases were collected for 5 min before each run. The HR, RPE, score of the perceived thirst scale (PTS), and score of the perceived abdominal discomfort scale (PAS) were also recorded. A 10-point visual analog scale was used for the PTS and PAS. A score of 1 indicated "Not Thirsty" and "No discomfort" respectively, whereas a score of 10 indicated "Very Very Thirsty" and "Very Very discomfort" respectively. After the standing gas collection, the pre-exercise capillary blood samples were collected to measure the levels of hemoglobin (Reflotron ${ }^{\circledR}$ System, Boehringer Mannheim, Germany), hematocrit (Clay Adams, Autocrit Ultra 3, Englewood, NJ, USA), blood glucose (Model 1502, YSI, Yellow Springs, OH, USA), blood lactate (Model 1502, YSI), and osmolality (Vapor Pressure Osmometer 5520, Wescor Inc., Logan, UT, USA). Further capillary samples were taken during exercise to measure the concentrations of blood glucose and lactate. At the end of the exercise, the capillary blood samples were obtained again.

After the collection of the baseline measurements, a 5-min standardized warm-up began at a running speed corresponding to $60 \%$ of $\mathrm{VO}_{2}$ max. The expired gas was collected during the last minute, and the HR, RPE, PTS, and PAS were recorded. The treadmill speed was then adjusted to a pace equivalent to $70 \%$ of $\mathrm{VO}_{2 \max }$ following the warm-up. During both trials, the subjects were required to run for as long as possible. Their endurance capacity was measured as the exercise time to volitional fatigue. Volitional fatigue was defined as the point at which the subject could no longer maintain the required running speed. To ensure maximal effort during each trial, the subjects were given strong verbal encouragement throughout the run; this encouragement was given only by blinded experimenters who were unaware of 
which treatment had been administered. No external time clues (i.e., clocks or radio) were provided, so the participants were not aware of their performance time until every experimental test had been concluded.

The expired air samples were collected over $2 \mathrm{~min}$ at 15-min intervals and during the last min before perceived exhaustion during the trials. The oxygen $\left(\mathrm{O}_{2}\right)$ and carbon dioxide $\left(\mathrm{CO}_{2}\right)$ content were measured with a paramagnetic $\mathrm{O}_{2}$ analyzer and a $\mathrm{CO}_{2}$ analyzer (MOXUS modular metabolic system, AEI Technologies Inc., Pittsburgh, PA, USA). Both analyzers were calibrated against a "gold standard" reference gas immediately before each series of gas analyses. The $\mathrm{VO}_{2}$ and $\mathrm{VCO}_{2}$ were determined from the gas analyses, and the RER was calculated. The rates of $\mathrm{CHO}$ and fat oxidation were calculated from $\mathrm{VO}_{2}$ and $\mathrm{VCO}_{2}$ values using stoichiometric equations [19]. The method of collection and analysis of expired air samples has been previously described [20]. The RPE, PTS, and PAS were also recorded every $15 \mathrm{~min}$ and during the last minute before perceived exhaustion during the experimental trials.

\subsection{Statistical Analysis}

The data analysis was performed with SPSS software (version 16.0). A two-way analysis of variance (ANOVA) for repeated measures (Trial $\times$ Time) was used to analyze the changes in the CHO oxidation rate, RER, concentrations of blood glucose and lactate, HR, and some subjective measures, such as RPE, PTS, and PAS. Significant differences between means were identified using the Tukey post hoc test. A paired $t$-test was used to analyze the differences in the nutritional data, pre-exercise BM, fluid ingestion, relative exercise intensity, and time to exhaustion between the two trials. The level of significance ( $p$ value) was accepted at 0.05 . The results are reported as mean $\pm \mathrm{SEM}$.

\section{Results}

No significant differences were found in the habitual dietary intake between the subjects in the CES and PL trials (Energy: $1736 \pm 172$ vs. $1582 \pm 172$ Kcal; CHO: $52.9 \% \pm 3.5 \%$ vs. $54.7 \% \pm 3.1 \%$; Protein: $20.0 \% \pm 1.9 \%$ vs. $19.5 \% \pm 0.6 \%$; Fat: $34.0 \% \pm 3.9 \%$ vs. $35.5 \% \pm 3.3 \%, p>0.05)$. The subject's pre-exercise BM was also similar between the two trials (CES vs. PL: $47.8 \pm 0.8$ vs. $47.9 \pm 0.7 \mathrm{~kg}$, $p>0.05$ ). Over the course of the run, the subjects drank a total volume of $644 \pm 75 \mathrm{ml}$ of fluid during the CES trial and $593 \pm 66 \mathrm{~mL}$ in the PL trial $(p>0.05)$. The participants ingested a total of $38.6 \pm 4.5 \mathrm{~g}$ of CHO during the CES trial.

\subsection{Exercise Time to Exhaustion}

The relative exercise intensities were similar on both occasions (CES vs. PL: $71 \% \pm 2 \% v s .69 \% \pm 2 \%$ of $\mathrm{VO}_{2 \max }, p>0.05$ ). The exercise time to exhaustion was significantly longer (by approximately $16 \%$ ) during the CES trial than during the placebo trial (CES vs. PL: $106.2 \pm 9.4$ vs. $91.6 \pm 5.9$ min, $p<0.05)$. 


\subsection{Expired Air Analysis}

The pattern of change in the RER was similar in both trials $(p=0.385)$. The overall CHO oxidation rates during exercise were also similar in both trials (CES vs. PL: $1.2 \pm 0.1 \mathrm{~g} \cdot \mathrm{min}^{-1} v s .1 .4 \pm 0.1 \mathrm{~g} \cdot \mathrm{min}^{-1}$, $p=0.116)$. Only a transient decrease in the $\mathrm{CHO}$ oxidation rate was found in the $60^{\text {th }}$ minute of the CES trial (Figure 1).

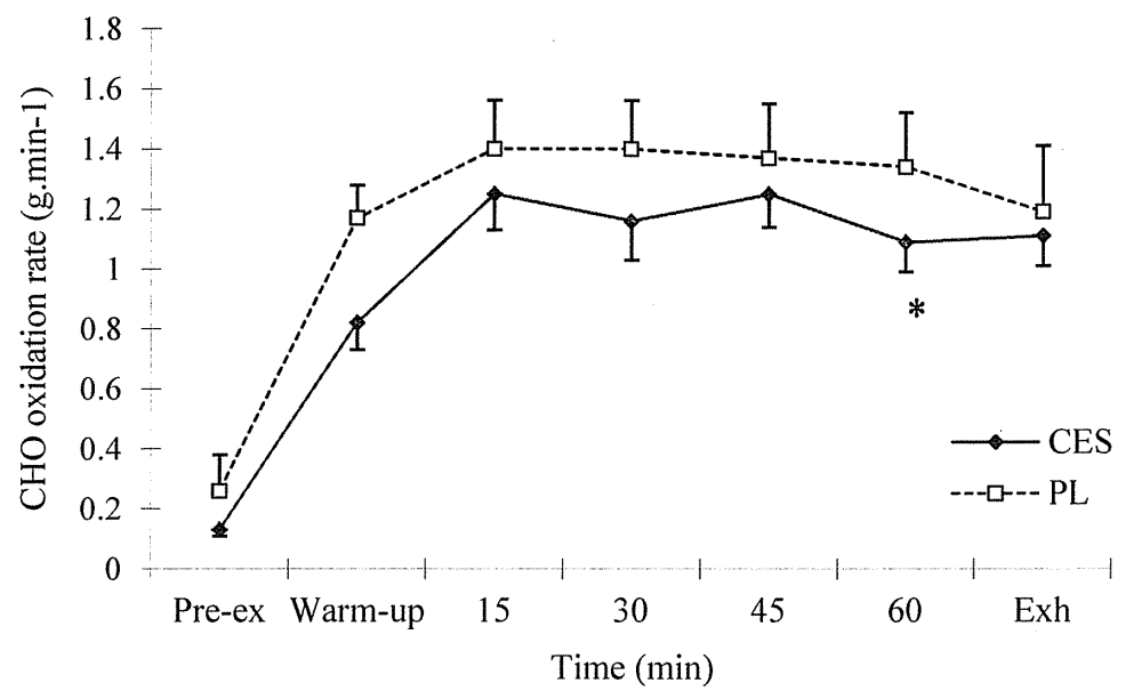

Figure 1. $\mathrm{CHO}$ oxidation rate $\left(\mathrm{g} \cdot \mathrm{min}^{-1}\right)$ during treadmill running. Values (mean $\left.\pm \mathrm{SEM}\right)$ are for placebo (PL) and carbohydrate-electrolyte solution (CES) trials. * CES $v s$. PL, $p<0.05$.

\subsection{Blood Sampling Analysis}

The serum osmolality was similar between the two conditions (CES vs. PL: $288.4 \pm 2.1$ vs. $\left.285.9 \pm 3.4 \mathrm{mOsm} \cdot \mathrm{l}^{-1}, p>0.05\right)$. The blood glucose levels $(\mathrm{F}=2.357 ; p=0.060)$ and blood lactate concentrations $(\mathrm{F}=0.947 ; p=0.492)$ were similar between the CES and PL trials (Figure 2A,B). However, at $45 \mathrm{~min}$ during exercise, the plasma glucose concentration was higher in the CES trial than in PL trial. Furthermore, the plasma glucose concentration increased gradually from the 15-min mark of the CES trial.

\subsection{Heart Rate, Ratings of Perceived Exertion, Thirst and Abdominal Discomfort}

There were no differences in the patterns of change in the exercising HR and perceptual variables between the two trials (Table 1), including RPE $(\mathrm{F}=1.767 ; p=0.129)$, PTS $(\mathrm{F}=1.161 ; p=0.347)$, and PAS $(\mathrm{F}=0.046 ; p=0.999)$. 

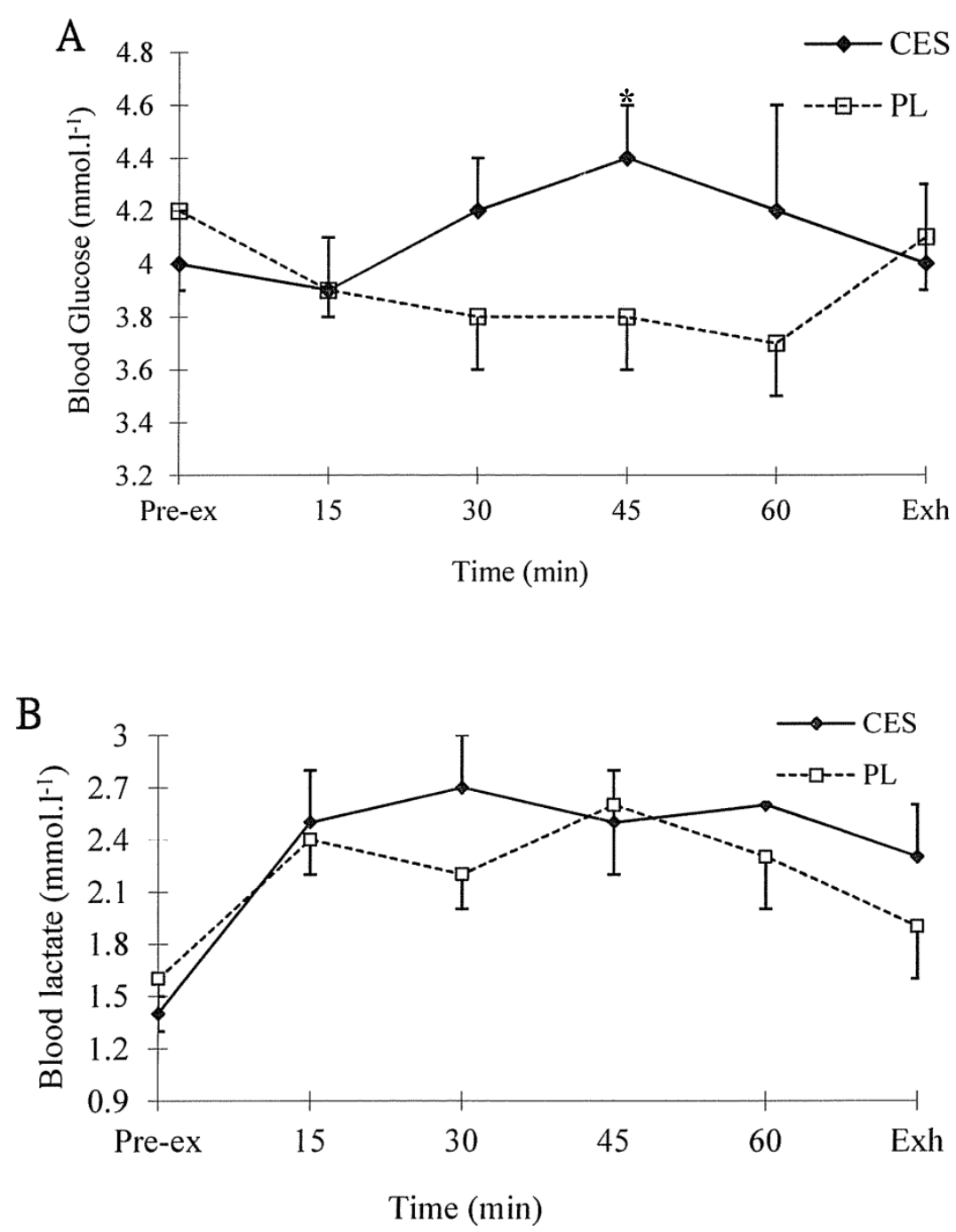

Figure 2. Blood glucose (A) and lactate (B) concentration during treadmill running. Values (mean \pm SEM) are for placebo (PL) and carbohydrate-electrolyte solution (CES) trials. * CES vs. PL, $p<0.05$. 
Table 1. Summary of the heart rate, rate of perceived exertion (RPE), perceived thirst scale (PTS) and perceived abdominal discomfort scale (PAS) during running in the carbohydrate-electrolyte solution (CES) and placebo (PL) trials, values are presented as mean \pm SEM.

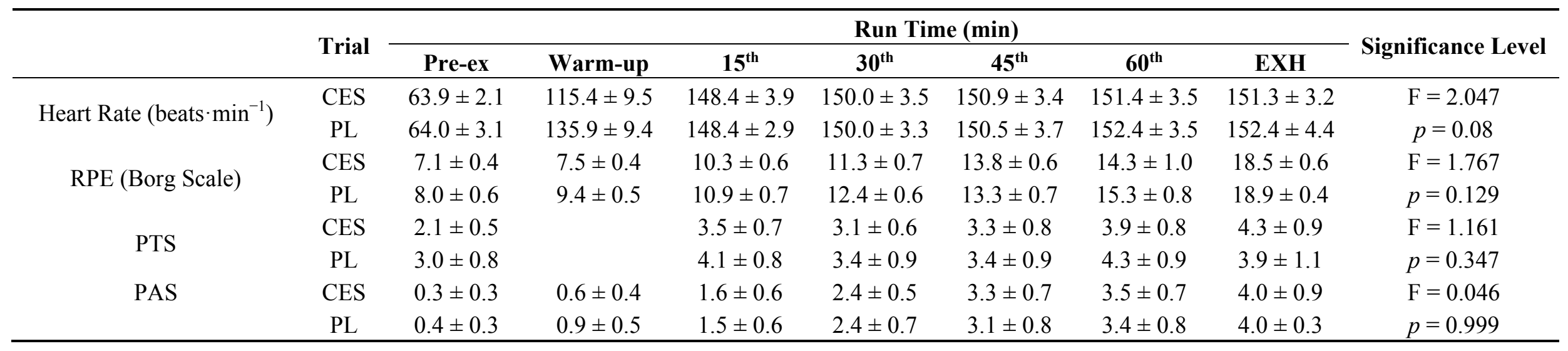




\section{Discussion}

The main finding of the present study was that the overall endurance time was increased with the ingestion of CES compared with placebo solution in active females.

It has been reported that $\mathrm{CHO}$ ingestion during endurance exercise improves exercise performance in male subjects in both exercise to exhaustion and time-trial protocols [6]. Although several studies in females found improved performance after $\mathrm{CHO}$ consumption [10,11], not all studies have supported this conclusion [9]. In this specific study, no improvement was found in performance during a $24.2-\mathrm{km}$ time trial when CHOs were consumed during exercise [9]. However, in another study that included $2 \mathrm{~h}$ of steady cycling plus a time-trial exercise protocol, the authors found that the ingestion of glucose improved exercise performance compared with the ingestion of a placebo [11]. In the present study, an improvement of approximately $16 \%$ was gained in the CES trial compared to the PL trial, which was slightly higher than the figure found in a previous study with a cycling to exhaustion exercise protocol [10]. The different performance measurement protocols may be one factor that contributes to the inconsistent findings, because performance as determined by different methods may vary widely [21] and different physiological responses were found between cycling and running tests [22]. Although it has been argued that exercise to exhaustion protocol has relatively poor test-retest reliability [21], it is still one commonly used method to test the endurance capacity. The improved endurance capacity may come from the increased exogenous $\mathrm{CHO}$ consumption during exercise, which could maintain the blood glucose concentration and attenuate the rate of glycogenolysis, thereby sparing muscle glycogen stores and delaying the onset of fatigue [8]. In the present study, although no statistical difference was found in the blood glucose concentrations between the two trials, there was a trend $(p=0.06)$ toward higher blood glucose concentrations in the CES trial than in the PL trial. Furthermore, the blood glucose concentration was higher at 45 min of exercise in the CES trial than in the PL trial (Figure 2). In addition, the CHO oxidation rate during the endurance run did not differ significantly between the two trials (Figure 1). These results indicate that the increased exogenous $\mathrm{CHO}$ oxidation may not be the major reason for the improved endurance capacity in the present study. Some other factors, such as an attenuation of central fatigue, may also play a role in the improved endurance capacity [7]. Therefore, one limitation of the present study is that the exact mechanism and/or ideal composition and dosage of CES could not be identified. However, it is obvious that commercially available CES is beneficial to the moderate intensity endurance capacity of active females during the follicular phases of the menstrual cycle.

It has been found that the phase of menstrual cycle does not alter the effects of CHO supplementation on performance [10]. In that study, the decrease in the plasma glucose level was attenuated during both the luteal and follicular phases of the menstrual cycle when CHOs were consumed during exercise. On the other hand, another study [11] found that the substrate metabolism and exercise performance were affected by the different phases of the menstrual cycle. The $\mathrm{CHO}$ contribution and performance were greater in the follicular phase than those in the luteal phase. The ingestion of glucose during exercise may minimize these effects. Therefore, the findings of the present study further suggest that $\mathrm{CHO}$ consumption may improve endurance capacity during the follicular phase. Some studies have found differences according to gender [23,24] in the metabolic responses of $\mathrm{CHO}$ ingested during exercise, whereas others did not [25]. Comparing with male, female may prefer to use more lipids but less CHO during endurance exercise [12]. This may be one reason that no difference was found in CHO oxidation 
rate between two trials in the present study. More studies are obviously needed to clarify whether the conclusions drawn from male subjects can be generalized to female subjects, especially in consideration of the menstrual cycle.

Another factor that may cause fatigue during endurance exercise is dehydration. Even moderate dehydration has been suggested to have negative effects on endurance performance [2,15]. However, in the present study, the hydration status did not seem to differ between the two trials. The finding was supported by the fact that no difference was found in the serum osmolality between the two trials. The regular ingestion of fluids is strongly recommended during endurance exercise [2]. Most commercially available CES are considered to be appropriate for endurance exercise, but the optimal composition is still unclear [2]. A CES not only supplies energy to spare the body's limited CHO stores, but also replaces electrolytes that are lost in sweat [26]. The ingestion of sodium-containing solutions during exercise has been found to be more effective in preventing a decrease in the plasma volume than the ingestion of pure water [27]. A high sodium content has also been suggested to make the drinks more effective in rehydrating athletes [28]. Therefore, although the potential effects of electrolytes on endurance capacity could not be excluded in the present study, it seems that the improvement of exercise performance should not be the different hydration level between CES and PL trials.

The HR and blood lactate concentrations were kept constant during the exercise in both trials. This indicated that no dehydration occurred and that the internal loads were similar between the conditions. The RPE increased gradually with both treatments and in the same pattern. In addition, the PTS and PAS of the subjects in the present study remained low and were similar in both trials, which indicate that neither CES nor PL caused abdominal problems during exercise.

Another limitation of the present study is that capillary blood samples were collected to measure the blood glucose and lactate concentrations, which can provide only limited information about the metabolic responses during exercise between the two trials. Further studies are still needed to clarify the potential mechanisms behind the improved running performance in active females who consume a CES during endurance exercise.

\section{Conclusions}

In conclusion, the results of the present study further confirm that CES supplementation improves the moderate intensity endurance capacity of active females during the follicular phases of the menstrual cycle. However, the exogenous $\mathrm{CHO}$ oxidation does not seem to explain the improved capacity after CES supplementation.

\section{Acknowledgments}

The authors would like to thank all the participants in the present study.

\section{Author Contributions}

Feng-Hua Sun and Stephen Heung-Sang Wong were responsible for designing the study, conducting analyses, interpreting the output of analyses and preparing the manuscript for submission. Shi-Hui Chen and Tsz-Chun Poon interpreted data and revised the manuscript for intellectual content. 


\section{Conflicts of Interest}

The authors declare no conflict of interest.

\section{References}

1. Coyle, E.F. Substrate utilization during exercise in active people. Am. J. Clin. Nutr. 1995, 61, S968-S979.

2. Sawka, M.N.; Burke, L.M.; Eichner, E.R.; Maughan, R.J.; Montain, S.J.; Stachenfeld, N.S. Exercise and fluid replacement. Med. Sci. Sports Exerc. 2007, 39, 377-390.

3. Tsintzas, K.; Williams, C.; Wilson, W.; Burrin, J. Influence of carbohydrate supplementation early in exercise on endurance running capacity. Med. Sci. Sports Exerc. 1996, 28, 1373-1379.

4. Jeukendrup, A.E. Carbohydrate intake during exercise and performance. Nutrition 2004, 20, 669-677.

5. Rodriguez, N.R.; di Marco, N.M.; Langley, S. American College of Sports Medicine position stand. Nutrition and athletic performance. Med. Sci. Sports Exerc. 2009, 41, 709-731.

6. Temesi, J.; Johnson, N.A.; Raymond, J.; Burdon, C.A.; O’Connor, H.T. Carbohydrate ingestion during endurance exercise improves performance in adults. J. Nutr. 2011, 141, 890-897.

7. Karelis, A.D.; Smith, J.E.W.; Passe, D.H.; Péronnet, F. Carbohydrate administration and exercise performance: What are the potential mechanisms involved? Sports Med. 2010, 40, 747-763.

8. Cermak, N.; van Loon, L.C. The use of carbohydrates during exercise as an ergogenic aid. Sports Med. 2013, 43, 1139-1155.

9. Andrews, J.L.; Sedlock, D.A.; Flynn, M.G.; Navalta, J.W.; Ji, H. Carbohydrate loading and supplementation in endurance-trained women runners. J. Appl. Physiol. 2003, 95, 584-590.

10. Bailey, S.P.; Zacher, C.M.; Mittleman, K.D. Effect of menstrual cycle phase on carbohydrate supplementation during prolonged exercise to fatigue. J. Appl. Physiol. 2000, 88, 690-697.

11. Campbell, S.E.; Angus, D.J.; Febbraio, M.A. Glucose kinetics and exercise performance during phases of the menstrual cycle. Am. J. Physiol. Endocrin. Metab. 2001, 281, E817-E825.

12. Tarnopolsky, L.J.; MacDougall, J.D.; Atkinson, S.A.; Tarnopolsky, M.A.; Sutton, J.R. Gender differences in substrate for endurance exercise. J. Appl. Physiol. 1990, 68, 302-308.

13. Pfeiffer, B.; Stellingwerff, T.; Zaltas, E.; Jeukendrup, A.E. Oxidation of solid versus liquid CHO sources during exercise. Med. Sci. Sports Exerc. 2010, 42, 2030-2037.

14. Pfeiffer, B.; Stellingwerff, T.; Zaltas, E.; Jeukendrup, A.E. CHO oxidation from a CHO gel compared with a drink during exercise. Med. Sci. Sports Exerc. 2010, 42, 2038-2045.

15. Maughan, R.J. Impact of mild dehydration on wellness and on exercise performance. Eur. J. Clin. Nutr. 2003, 57, S19-S23.

16. Montain, S.J.; Coyle, E.F. Influence of graded dehydration on hyperthermia and cardiovascular drift during exercise. J. Appl. Physiol. 1992, 73, 1340-1350.

17. Nose, H.; Mack, G.W.; Shi, X.R.; Nadel, E.R. Role of osmolality and plasma volume during rehydration in humans. J. Appl. Physiol. 1988, 65, 325-331. 
18. Wong, S.H.S.; Williams, C.; Adams, N. Effects of ingesting a large volume of carbohydrate-electrolyte solution on rehydration during recovery and subsequent exercise capacity. Int. J. Sport Nutr. Exerc. Metab. 2000, 10, 375-393.

19. Frayn, K.N. Calculation of substrate oxidation rates in vivo from gaseous exchange. J. Appl. Physiol. 1983, 55, 628-634.

20. Wong, S.H.S.; Williams, C.; Simpson, M.; Ogaki, T. Influence of fluid intake pattern on short-term recovery from prolonged, submaximal running and subsequent exercise capacity. J. Sports Sci. 1998, $16,143-152$.

21. Currell, K.; Jeukendrup, A.E. Validity, reliability and sensitivity of measures of sporting performance. Sports Med. 2008, 38, 297-316.

22. Gore, C.J.; Scroop, G.C.; Marker, J.D.; Catcheside, P.G. Plasma volume, osmolarity, total protein and electrolytes during treadmill running and cycle ergometer exercise. Eur. J. Appl. Physiol. Occup. Physiol. 1992, 65, 302-310.

23. Horton, T.J.; Grunwald, G.K.; Lavely, J.; Donahoo, W.T. Glucose kinetics differ between women and men, during and after exercise. J. Appl. Physiol. 2006, 100, 1883-1894.

24. Tremblay, J.; Peronnet, F.; Massicotte, D.; Lavoie, C. Carbohydrate supplementation and sex differences in fuel selection during exercise. Med. Sci. Sport. Exerc. 2010, 42, 1314-1323.

25. Wallis, G.A.; Dawson, R.; Achten, J.; Webber, J.; Jeukendrup, A.E. Metabolic response to carbohydrate ingestion during exercise in males and females. Am. J. Physiol. Endocrin. Metab. 2006, 290, E708-E715.

26. IOC. IOC consensus statement on sports nutrition 2010. J. Sports Sci. 2011, 29, S3-S4.

27. Candas, V.; Libert, J.P.; Brandenberger, G.; Sagot, J.C.; Amoros, C.; Kahn, J.M. Hydration during exercise: Effects on thermal and cardiovascular adjustments. Eur. J. Appl. Physiol. 1986, 55, $113-122$.

28. Shirreffs, S.M.; Aragon-Vargas, L.F.; Keil, M.; Love, T.D.; Phillips, S. Rehydration after exercise in the heat: A comparison of 4 commonly used drinks. Int. J. Sport Nutr. Exerc. Metab. 2007, 17, $244-258$.

(C) 2015 by the authors; licensee MDPI, Basel, Switzerland. This article is an open access article distributed under the terms and conditions of the Creative Commons Attribution license (http://creativecommons.org/licenses/by/4.0/). 